\title{
The role of diffusion and perfusion magnetic resonance imaging in differentiation of haemangioblastomas and pilocytic astrocytomas
}

\author{
Małgorzata Neska-Matuszewska, Anna Zimny, Joanna Bladowska, Anna Czarnecka, Marek Sąsiadek \\ Department of General and Interventional Radiology and Neuroradiology, Wroclaw Medical University, Poland
}

\section{Abstract}

\begin{abstract}
Purpose: Haemangioblastomas (HABLs) and pilocytic astrocytomas (PAs) are brain tumours presenting similar appearance and location in conventional magnetic resonance (MR) imaging. The purpose of our study was to determine whether a detailed analysis of diffusion (DWI) and perfusion (PWI) characteristics can be useful in preoperative differentiation of these tumours.

Material and methods: The study group consisted of biopsy proven six HABLs and six PAs, which underwent preoperative standard MR examinations including PWI and DWI. In PWI relative cerebral blood volume (rCBV) and the shape of perfusion curves (parameters of peak height - $\mathrm{rPH}$ and percentage of signal recovery - rPSR) were analysed. All perfusion parameters were measured for the entire tumour core (mean $\mathrm{rCBV}$, mean $\mathrm{rPH}$, mean $\mathrm{rPSR}$ ) and in regions with maximal values ( $\max \mathrm{rCBV}$, $\max \mathrm{rPH}$, max $\mathrm{rPSR}$ ). In DWI parameters of apparent diffusion coefficient (ADC) from the entire tumour core (mean ADC) and in regions with minimal values (min ADC) were evaluated.

Results: Compared to PAs, HABLs presented significantly higher rCBV and rPH values and lower mean rPSR value. PAs showed significantly lower $\mathrm{rCBV}$ and $\mathrm{rPH}$ values and higher mean $\mathrm{rPSR}$ value. Mean rCBV showed no overlap in the values between HABLs and PAs, and thus it provided the highest accuracy in differentiating between them. Max rPSR, mean ADC, and min ADC did not show any significant differences.
\end{abstract}

Conclusions: High rCBV values and deep perfusion curves with only partial return to the baseline are characteristic features of HABLs differentiating them from PAs, which show lower rCBV values and perfusion curves overshooting the baseline. Diffusion parameters are not useful in differentiation of these tumours.

Key words: PWI, DWI, haemangioblastoma, pilocytic astrocytoma.

\section{Introduction}

Cerebellar haemangioblastomas (HABLs) and pilocytic astrocytomas (PAs) are two types of brain tumours that show similar appearance in conventional magnetic resonance (MR) imaging, typically presenting as a cyst with an associated mural nodule. Both of them are usually clinically benign and are classified as grade I by the World Health Organisation (WHO). The patient's age is often helpful in distinguishing these tumours associating HABLs with adult patients and PAs with the paediatric population, but it is not always reliable [1,2]. Nevertheless, there is a group of young adults in whom both types of tumours may occur. Haemangioblastomas account for $1-2.5 \%$ of intracranial tumours and $7-12 \%$ of posterior fossa neoplasms in adult patients $[3,4]$. Pilocytic astrocytomas are the most common paediatric central nervous system (CNS) gliomas and one of the most common paediatric cerebellar tumours representing $60 \%$ of all astrocytomas in children [2]. They may also occur in adults, reaching in this group 2.3-6\% of all brain tumours [1].

Correspondence address:

Małgorzata Neska-Matuszewska, Department of General and Interventional Radiology and Neuroradiology, Wroclaw Medical University, 213 Borowska St.,

50-556 Wroclaw, Poland, phone/fax: +48 600231 210, e-mail: neskamatuszewska@gmail.com

Authors' contribution:

A Study design · B Data collection · C Statistical analysis · D Data interpretation · E Manuscript preparation · F Literature search · G Funds collection 
Morphologically, both HABLs and PAs may show heterogeneous appearance; mainly cystic but also solid-cystic or solid lesions are observed $[3,4]$.

Both tumours are potentially curable by surgical resection and are associated with longer survival [5]. However, their histopathological structure has an impact on different clinical and surgical approaches. Since HABLs represent capillary-rich neoplasms of the CNS with large networks of tightly packed blood vessels lined by endothelial cells on a background of abundant connective tissue [6], their surgical resection should encompass the whole tumour capsule, otherwise it could lead to high mortality and morbidity rates due to the complication of excessive bleeding [3,7]. Sometimes preoperative embolisation of the feeding arteries may reduce the tumour blood supply, which can decrease intraoperative haemorrhage [8]. On the other hand, PAs, as less vascularised tumours, are composed of a variable combination of loose connective glial tissue, compacted piloid tissue, numerous vacuoles, microcysts, and sometimes macrocysts [6], so their potential complete resection is easier and safer.

As presented above, the reliable preoperative differentiation between HABLs and PAs is important but, in many cases, challenging in the standard MR imaging.

Advanced MR techniques such as perfusion-weighted imaging (PWI) and diffusion-weighted imaging (DWI) may add functional information and help in the in vivo characterisation of these two tumour types.

Perfusion-weighted imaging is the method that brings information on cerebral physiology at the capillary level (microvasculature) $[9,10]$. Among the PWI techniques dynamic susceptibility contrast (DSC) MR imaging is the most often used. DSC MRI provides maps of cerebral blood volume $(\mathrm{CBV})$ and noninvasive measurements of relative cerebral blood volume (rCBV) $[9,10]$ as well as perfusion curves, which give an insight into the dynamics of the first pass of the contrast material through the microvasculature. Analysis of perfusion curves has been reported to be very useful in the evaluation of brain tumours $[9,10]$, in some cases more important than analysis of the CBV maps [10]. Two haemodynamic parameters derived from perfusion curves: the peak height $(\mathrm{PH})$ and the percentage of signal intensity recovery (PSR), have been shown to provide important, additional information regarding tumor capillary volume and capillary permeability respectively [10].

On the other hand, DWI is a sensitive tool that allows quantifying of physiological alterations in water diffusion, which result from microscopic structural changes not detectable with anatomical MR imaging. Water diffusion can be measured with a parameter of apparent diffusion coefficient (ADC) $[11,12]$. Diffusivity of water depends primarily on the presence of microscopic structural barriers in tissue such as membranes of cell bodies, axons, and myelin sheaths but also viscosity of adjacent structures that can alter the random motion of water molecules. Highly cellular tumours show areas of restricted diffusion with low ADC values, thus ADC is regarded as a marker of tumour cellularity $[11,12]$.

In the literature there are a few reports that compare HABLs and PAs in advanced MR techniques, but none of them focuses on both DWI and PWI together in one study. The purpose of our study was to determine whether a detailed analysis of diffusion and perfusion characteristics with the evaluation of perfusion curves is useful in preoperative differentiation of these tumours.

\section{Material and methods}

The study group consisted of 12 solitary brain tumours, which were selected from a cohort of 1210 subjects with CNS tumours evaluated with T2*DSC perfusion and diffusion MR in our institution between January 2010 and July 2017. Our material was subdivided into biopsy proven six HABLs (three men, three women) aged 32-73 years (mean age: 52 years) and six PAs (one man, five women) aged 14-67 years (mean age: 36 years). All tumours were solitary lesions located either infra- or supratentorially (Table 1).

\section{Data acquisition}

All examinations were performed on 1.5 T MR scanner (Signa Hdx, GE Medical Systems) using a 16-channel HNS (head-neck-spine) coil.

Before contrast administration a standard MR examination was carried out including axial T1-weighted images, axial, coronal, and sagittal T2-weighted images as well as axial FLAIR images followed by DWI and post-contrast DSC T2*-perfusion and 3D T1-weighted imaging.

During the whole MR examination the patients were instructed to keep their eyes closed. No sedation or anaesthesia were used in any of the patients.

Perfusion examination was performed with a dynamic susceptibility contrast (DSC) method using fast echoplanar T2*-weighted gradient echo sequence with the following parameters: $\mathrm{TR}=1.900 \mathrm{~ms}, \mathrm{TE}=80 \mathrm{~ms}, \mathrm{FOV}=30 \mathrm{~cm}$, matrix $=192 \times 128$, slice thickness $=8 \mathrm{~mm}$ without spacing, NEX - 1.0. Ten seconds after the start of the image acquisition a bolus of $1.0 \mathrm{~mol} / \mathrm{l}$ gadobutrol formula (Gadovist, Bayer Health Care, Germany) in a dose of $0.1 \mathrm{ml} / \mathrm{kg}$ of body weight was injected via a 20 -gauge catheter placed in the antecubital vein. Contrast was administered with an automatic injector (Medrad) at a rate of $5 \mathrm{ml} / \mathrm{s}$ and was followed by a saline bolus $(20 \mathrm{ml}$ at $5 \mathrm{ml} / \mathrm{s}$ ). The whole perfusion imaging lasted $1 \mathrm{~min} 26 \mathrm{~s}$, in which sets of images from 13 axial slices were obtained before, during and after contrast injection. After PWI a post-contrast $\mathrm{T} 1$-weighted $3 \mathrm{D}$ sequence was performed using contrast bolus administered earlier for the perfusion examination. No contrast agent was administered before DSC perfusion MR imaging.

Diffusion imaging was performed using a transverse single-shot echoplanar diffusion-weighted sequence with 
Table 1. Demographic and imaging characteristics of haemangioblastomas and pilocytic astrocytomas

\begin{tabular}{|c|c|c|c|c|c|}
\hline Patient & Age (years) & Sex & Location & Morphology & Enhancement \\
\hline \multicolumn{6}{|c|}{ Haemangioblastomas } \\
\hline 1 & 73 & Female & Cerebellum & Cystic with mural nodule & Homogenous \\
\hline 2 & 49 & Female & Cerebellum & Solid & Homogenous \\
\hline 3 & 37 & Male & Cerebellum & Cystic with mural nodule & Homogenous \\
\hline 4 & 63 & Male & Cerebellum & Solid with necrosis & Inhomogenous \\
\hline 5 & 57 & Female & Suprasellar region & Solid & Homogenous \\
\hline 6 & 32 & Male & Peripheral, in the right temporal lobe & Solid with necrosis & Inhomogenous-peripheral \\
\hline \multicolumn{6}{|c|}{ Pilocytic astrocytomas } \\
\hline 1 & 14 & Female & Cerebellum & Cystic-solid with necrosis & Inhomogenous \\
\hline 2 & 63 & Female & Cerebellum & Solid & Homogenous-peripheral \\
\hline 3 & 25 & Female & Cerebellum & Solid & Inhomogenous \\
\hline 4 & 23 & Female & Pperipheral, in the right frontal lobe & Solid & Homogenous \\
\hline 5 & 67 & Male & Peripheral, in the left parietal lobe & Solid with necrosis & Inhomogenous-peripheral \\
\hline 6 & 26 & Female & Suprasellar region & Solid with necrosis & Inhomogenous \\
\hline
\end{tabular}

the following parameters: $\mathrm{TE}=89.9 \mathrm{~ms}, \mathrm{TR}=8000 \mathrm{~ms}$, slice thickness $=5 \mathrm{~mm}, \mathrm{FOV}=26 \mathrm{~cm}$, matrix size $=128$ $\times 128$, NEX -1 , diffusion sensitive gradient $b=1000 \mathrm{~s} /$ $\mathrm{mm}^{2}$ in three basic orthogonal directions, scanning time: 42 seconds.

\section{Data postprocessing}

In all cases the morphological assessment of the lesions was performed on T1, T2-weighted, and post-contrast T1-weighted images using visual inspection.

The diffusion and perfusion weighted images were postprocessed using Functool software (ADW 4.4; GE Medical Systems).

\section{Perfusion weighted imaging analysis}

The analysis was based on evaluation of CBV parameters on CBV maps (Figure 1) as well as values of PH (peak height) and PSR (percentage of signal recovery) derived from perfusion curves. Measurements of CBV were per- formed by placing ROIs on the CBV maps fused with post-contrast T1-weighted images to accurately assess the tumour core (Figure 1). PSR and PH were calculated from the perfusion curves based on the following formulas:

$$
\begin{gathered}
\mathrm{PSR}=(\mathrm{S} 1-\mathrm{Smin}) / \mathrm{PH} \\
\mathrm{PH}=\mathrm{Smin}-\mathrm{S} 0,
\end{gathered}
$$

where: $S O$ - start of contrast passage, Smin - maximal drop of magnetic susceptibility, and $S 1$ - measurement after 24 seconds from Smin (Figure 1). All CBV, PH, and PSR values were normalised to values from the normal appearing white matter of the contralateral hemisphere to obtain relative values of all parameters: $\mathrm{rCBV}, \mathrm{rPH}, \mathrm{rPSR}$.

In each tumour measurements of mean values of all perfusion parameters for the whole tumour core (mean rCBV, mean $\mathrm{rPH}$, mean rPSR) and maximal values of these parameters (max $\mathrm{rCBV}$, $\max \mathrm{rPH}$, max rPSR) were assessed. Mean values for the whole tumour were obtained by manual outlining of the entire lesion on each slice (Figure 1) avoiding foci of haemorrhage or necrosis and calculating the arithmetical means from all the measured values. Maximal values were obtained by placing

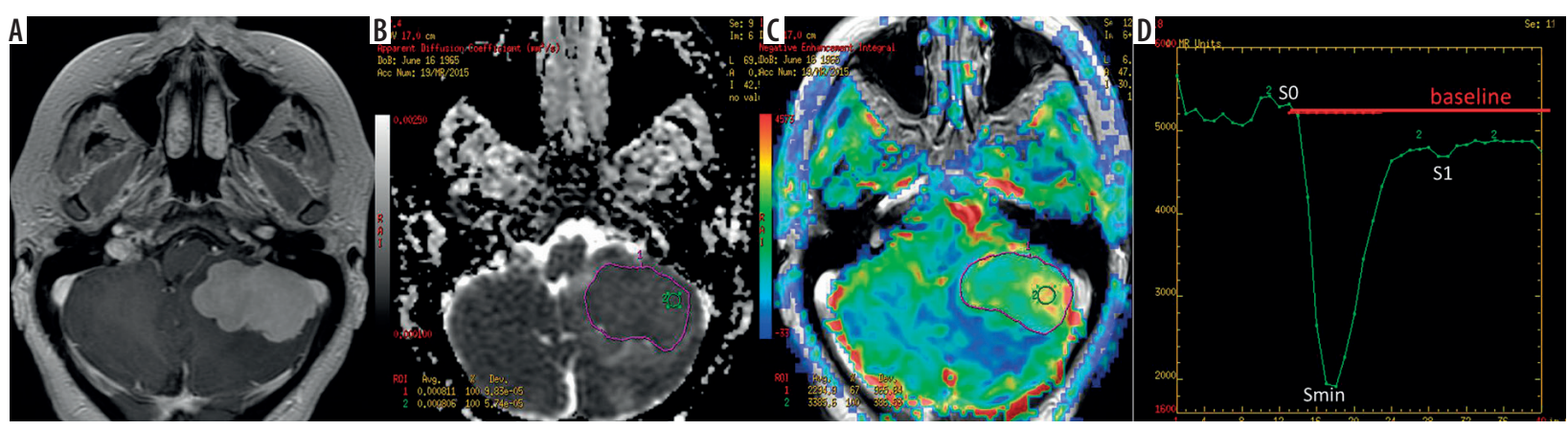

Figure 1. Haemangioblastoma in the left cerebellar hemisphere: (A) a post-contrast, T1-weighted image, (B) an ADC map, (C) a CBV map, (D) a perfusion signal intensity time curve. The ADC (B) and CBV (C) maps show placement of large irregular freehand ROls outlining the tumour core (purple) as well as small circular ROls within the big ROIs (green). The perfusion curve (D) shows haemodynamics of contrast agent during the first pass of a bolus through the brain vasculature, with the $x$-axis reflecting time in seconds and $y$-axis indicating signal intensity 
small ROIs $\left(40-60 \mathrm{~mm}^{2}\right.$ ) within several hot spots on each slice. The highest value from all ROIs was chosen as the tumoural maximal value (Figure 1).

\section{Diffusion-weighted imaging analysis}

Measurements of ADC for the entire tumour (mean $\mathrm{ADC}$ ) and measurements of minimum ADC (min ADC) were performed on ADC maps fused with post-contrast T1-weighted images. Similarly to perfusion evaluation, mean ADC values were obtained by manual outlining of the entire enhancing tumour core on each slice (Figure 1), avoiding foci of haemorrhage or necrosis, and then by calculating the arithmetical averages from all measured ADC values. Min ADC values were measured using small ROIs (40-60 $\mathrm{mm}^{2}$ ) located within the large freehand ROIs (Figure 1). The lowest value from all ROIs was chosen as the tumoural minimum ADC value described as min ADC.

\section{Statistical analysis}

All data obtained in the study were statistically analysed using the Statistica PL software package version 4.0. Quanti- tative perfusion and diffusion data were compared between HABL and PA groups using the student $t$-test, with a $p$ value below 0.05 set as the level of significance.

\section{Results}

Both HABLs and PAs were mainly located intra-axially in the cerebellum, although tumours in the supratentorial or extra-axial locations were also found (Table 1).

Based on standard MR examination HABLs and PAs presented variable morphology, both cystic and solid (Table 1, Figures 2 and 3). Compared to PAs, HABLs more often showed typical appearance of a cyst with a mural nodule as well as homogenous enhancement (Table 1, Figure 2). On the other hand, the majority of PAs were solid with areas of necrosis and inhomogeneous enhancement (Table 1, Figure 3).

All HABLs presented very high perfusion parameters of mean rCBV (6.44), max rCBV (12.01), mean rPH (5.31), max $\mathrm{rPH}$ (9.00) as well as low values of mean rPSR (0.21) and max rPSR (0.53), indicating only a slight return of the perfusion curve to the baseline level. Compared to HABLs, PAs showed significantly lower mean rCBV (2.14), max

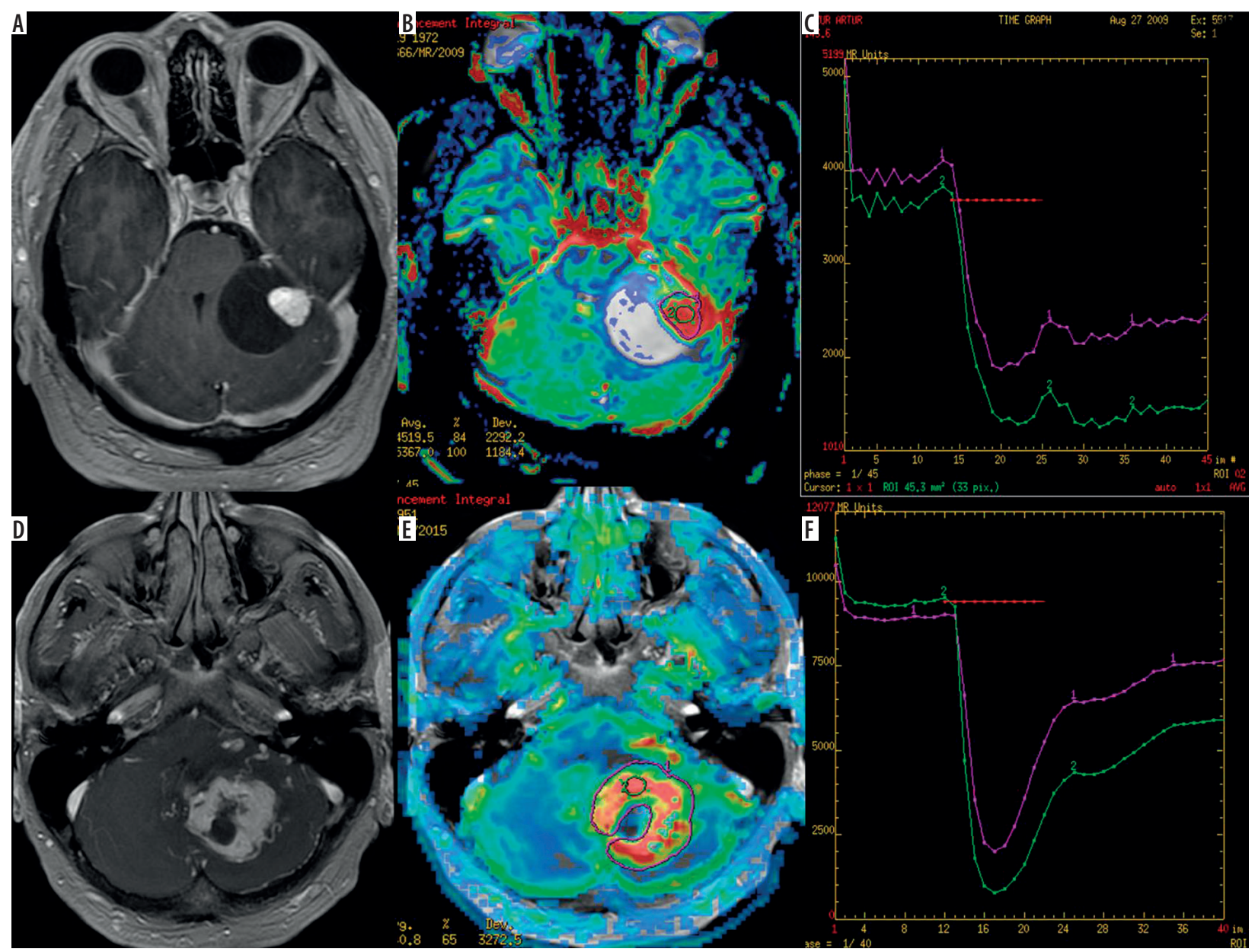

Figure 2. Two examples of haemangioblastomas in the cerebellum presenting as a cystic tumour with a mural nodule (A) and as a more solid tumour (D) on post-contrast T1-weighted images. CBV maps ( $B$ and $E$ ) show high perfusion values indicated by red colour while perfusion curves ( $C$ and $F)$ present only partial return to the baseline level (red lines) 


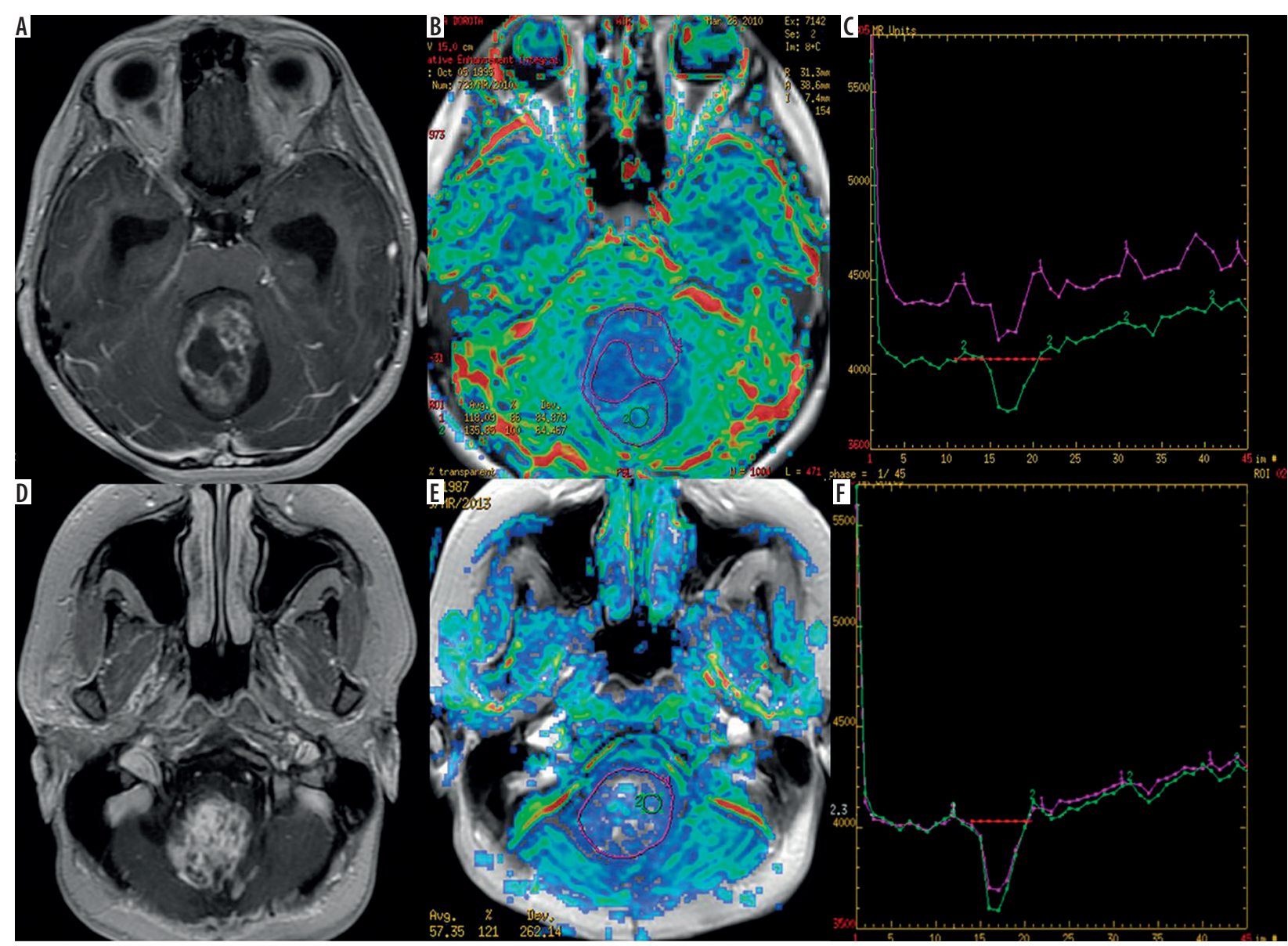

Figure 3. Two examples of pilocytic astrocytomas presenting as cystic-nodular (A) and solid tumours (D) on post-contrast T1-weighted images. (BV maps ( $B$ and $E$ ) show low perfusion values indicated by green and blue colours while perfusion curves ( $C$ and $F$ ) present return above the baseline level (red lines)

Table 2. Perfusion and diffusion measurements with the results of group comparisons indicated by $p$-value

\begin{tabular}{|c|c|c|c|c|c|c|c|c|}
\hline Patient & mean rCBV & $\max r C B V$ & mean rPH & $\max r \mathrm{PH}$ & mean rPSR & $\max$ rPSR & mean $A D C$ & $\min A D C$ \\
\hline \multicolumn{9}{|c|}{ Haemangioblastomas } \\
\hline 1 & 8.22 & 10.91 & 5.58 & 10.32 & 0.30 & 0.36 & 1.73 & 1.41 \\
\hline 2 & 5.1 & 9.10 & 5.1 & 9.65 & 0.30 & 0.98 & 0.81 & 0.76 \\
\hline 3 & 6.75 & 10.50 & 6.64 & 8.34 & 0.42 & 0.45 & 1.67 & 1.29 \\
\hline 4 & 9.13 & 22.07 & 5.90 & 9.65 & 0.20 & 0.70 & 1.68 & 1.31 \\
\hline 5 & 3.74 & 4.85 & 3.51 & 4.07 & -0.40 & 0.10 & 1.88 & 1.63 \\
\hline 6 & 5.71 & 14.61 & 5.13 & 11.99 & 0.46 & 0.56 & 1.11 & 0.69 \\
\hline Mean & 6.44 & 12.01 & 5.31 & 9.00 & 0.21 & 0.53 & 1.48 & 1.18 \\
\hline \multicolumn{9}{|c|}{ Pilocytic astrocytomas } \\
\hline 1 & 1.34 & 2.64 & 3.65 & 4.84 & 5.04 & 5.84 & 1.14 & 0.84 \\
\hline 2 & 2.67 & 3.54 & 2.51 & 3.21 & 0.97 & 1.00 & 0.84 & 0.65 \\
\hline 3 & 2.93 & 9.62 & 2.72 & 8.05 & 0.43 & 1.07 & 1.62 & 1.06 \\
\hline 4 & 1.27 & 1.82 & 2.11 & 2.38 & 1.83 & 1.84 & 1.05 & 0.94 \\
\hline 5 & 3.34 & 6.34 & 1.94 & 3.51 & 1.10 & 1.12 & 1.00 & 0.70 \\
\hline 6 & 1.30 & 1.81 & 1.82 & 2.07 & 1.71 & 1.74 & 1.05 & 0.82 \\
\hline Mean & 2.14 & 4.30 & 2.46 & 4.01 & 1.85 & 2.10 & 1.11 & 0.84 \\
\hline$p$-value & $0.0007^{*}$ & $0.01^{*}$ & $0.0002^{*}$ & $0.005^{*}$ & $0.03^{*}$ & 0.06 & 0.1 & 0.06 \\
\hline
\end{tabular}

*Statistically significant values 
rCBV (4.30), mean rPH (2.46), and max rPH (4.01) as well as significantly higher mean rPSR (1.85) and max rPSR (2.10) (Table 2). There were overlaps in parameters of max rCBV, mean $\mathrm{rPH}$, max $\mathrm{rPH}$, mean rPSR, and max rPSR but no overlap in the values of mean rCBV. Mean rCBV was found to be a parameter showing the highest accuracy in distinguishing these two analysed types of tumours (Table 2).

In DWI the values of mean $\mathrm{ADC}$ and min $\mathrm{ADC}$ were slightly higher in the HABL group (1.48 and 1.18, respectively) than in PAs (1.11 and 0.84, respectively). Moreover, there was an overlap in ADC values between HABLs and PAs (Table 2). No statistically significant differences were observed between average values of these parameters.

\section{Discussion}

Regarding the typical morphological appearance of both tumours as a cystic mass with enhancing mural nodule located in the cerebellum, our observations on conventional MR examination demonstrated many exceptions in both tumour location and morphology. In our study only one third of HABLs showed the typical morphological appearance as a cystic mass with a mural nodule located in the cerebellum (Table 1, Figure 2). Brundl et al. [3] analysed the morphology of 12 HABLs, reporting eight of them to have a typical appearance, which was $66.7 \%$ of the study group, whereas Kumar et al. [6] presented $50 \%$ of HABL tumours as typically appearing. Analysing PA cases, half of them were located extracerebellarly. The majority of PAs appeared solid and inhomogenous and only one case presented typically as a cystic mass with an enhancing nodule in the cerebellum (comprising only $\sim 16.7 \%$ of our PA cases) (Table 1, Figure 3). A study by Burkhard et al. [2] also analysed the location of PAs and showed that minority of cases (around $42 \%$ ) were located typically in the cerebellum. Moreover, Burkhard et al. [2] observed the tendency of PAs in children under 12 years old to be located typically in the cerebellum, contrary to adult patients. On the other hand, Stüer et al. [1] reported that only $21 \%$ of PAs presented a typical morphological appearance as a cystic mass with a mural nodule.

The variations and similarities in the presentation of HABLs and PAs reported in the literature $[1-3,6,8]$ coincide with our results and experience. It proves the importance of advanced MR techniques such as PWI and DWI, which can provide additional information on a microstructural level, which may in turn help in the differentiation between both tumour types in terms of their vascularisation and cellularity.

In our study we used PWI to evaluate tumour vascularisation and DWI to assess cellularity. Analysing perfusion studies we found significant differences between HABLs and PAs regarding both $\mathrm{rCBV}$ values derived from rCBV maps as well as shapes of perfusion curves.

Compared to PAs, HABLs showed significantly higher values of mean CBV and max CBV (Table 2, Figures 2 and 3), reflecting their high vascularisation. The parameter of rCBV was also evaluated by several other authors, and our results are in agreement with these studies $[6,8,13]$.

It must be stressed that in our study mean $\mathrm{rCBV}$ was the only perfusion parameter without any overlap in the values between two tumour types, with a range of 1.273.34 in PAs and 3.74-9.13 in HABLs, and therefore mean rCBV may be regarded as the parameter with the highest accuracy in distinguishing between both tumours.

In our opinion, analysis of perfusion curves may also be useful in differentiation between HABLs and PAs because these two tumour types show different shapes of signal intensity time curves. Our study revealed that in HABLs the signal drop described as $\mathrm{PH}$ was more pronounced, in contrast to PAs where the signal drop was relatively small (Table 2, Figures 2 and 3). The differences in both mean $\mathrm{rPH}$ and max $\mathrm{rPH}$ were statistically significant with $p$-values 0.0002 and 0.005 , respectively. Moreover, in HABLs signal recovery described as rPSR was less pronounced, with only partial return of the perfusion curve to the baseline level. In our study the majority of PA cases showed the signal time intensity curves crossing the baseline (overshooting) after the first pass; however, only mean rPSR values reached the level of significance.

Our results of the $\mathrm{PH}$ parameter are in agreement with the study by She et al. [14], who also showed statistically higher values of $\mathrm{PH}$ in HABLs than in PAs. On the other hand, the results of our PSR analysis only partially remain in agreement with the above mentioned study by She et al. [14], who found both max rPSR and mean rPSR to be higher in PA.

The differences in either $\mathrm{rCBV}$ or $\mathrm{rPH}$ values between HABLs and PAs may be explained easily by the distinct histological features of these two tumour types. As mentioned before, HABLs have abundant tumour vessels within lesions shown by high $\mathrm{rCBV}$ values and more pronounced signal drop reflected in $\mathrm{rPH}$ values, whereas PAs are sparsely vascularised in a wide interstitial space contributing to less pronounced perfusion curves and lower rCBV values $[6,14]$.

The explanation of PSR characteristics in both tumours is complicated because it is influenced by many factors, including the permeability of capillaries, the amount of contrast agent leakage, the rate of blood flow, as well as the size of extravascular space $[14,15]$. Moreover, the signal intensity is connected with the complex $\mathrm{T} 1$ - and $\mathrm{T} 2^{*}$-shortening effect from the accumulation of contrast agent in the interstitial space of lesion. When the T1-shortening effects, due to accumulation of contrast agent in the interstitial space, overwhelm the $\mathrm{T} 2^{*}$-shortening effects, the signal intensity increases and even exceeds the baseline level $[14,15]$. The significant difference in the mean rPSR between HABLs and PAs in this study may suggest that contrast agent leakage into the interstitial space in PAs is more pronounced than that in HABLs [8].

In our study DWI was proved to be a less specific and sensitive method in differentiation HABLs from PAs with 
no statistical significance. However, HABLs presented higher values of both mean ADC as well as min ADC in comparison to PAs (Table 2). Higher ADC values may be explained by a lower rate of cellularity in HABLs compared to PAs, and it may suggest the diagnosis of HABL in the posterior cranial fossa [16]. On the other hand, the results of $\mathrm{ADC}$ values in HABLs as well as in PAs appear similar to other benign tumours, which is consistent with previous reports $[8,17,18]$ and may help in differentiation from high grade tumours (Table 2).

\section{Conclusions}

Perfusion measurements are more useful than diffusion parameters in differentiation between HABLs and PAs.

High rCBV values and deep perfusion curves with only partial return to the baseline measured within the whole enhancing tumour part characterise HABLs and enable differentiation of them from PAs showing lower rCBV values and the curve overshooting the baseline.

The mean rCBV value showed no overlap in the values between HABLs and PAs, and thus it provided the highest accuracy in distinguishing between these tumour types.

Perfusion MR, as an easy to perform and rapid technique, should be routinely used in differentiation of brain focal lesions, including distinguishing HABLs from PAs.

\section{Acknowledgments}

The work was partially supported by Wroclaw Medical University grant No. ST-868.

\section{Conflict of interest}

The authors report no conflict of interest.

\section{References}

1. Stüer C, Vilz B, Majores M, et al. Frequent recurrence and progression in pilocytic astrocytoma in adults. Cancer 2007; 110: 2799-2808.

2. Burkhard C, Di Patre PL, Schuler D, et al. A population-based study of the incidence and survival rates in patients with pilocytic astrocytoma. J Neurosurg 2003; 98: 1170-1174.

3. Brundl E, Schodel P, Ullrich OW, et al. Surgical resection of sporadic and hereditary hemangioblastoma: Our 10-year experience and a literature review. Surg Neurol Int 2014; 5: 138.

4. Osborn AG, Hedlund G, Salzman KL. Osborn's Brain, Second Edition. Elsevier, Philadelphia 2017.

5. Koeller KK, Rushing EJ. From the archives of the AFIP: pilocytic astrocytoma: radiologic-pathologic correlation. Radiographics 2004; 24: 1693-1708.

6. Kumar VA, Knopp EA, Zagzag D. Magnetic resonance dynamic susceptibility-weighted contrast-enhanced perfusion imaging in the diagnosis of posterior fossa hemangioblastomas and pilocytic astrocytomas: Initial results. J Comput Assist Tomogr 2010; 34: 825-829.

7. Padhi S, Sarangi R, Challa S, et al. A 10-year retrospective study of hemangioblastomas of the central nervous system with reference to von Hippel-Lindau (VHL) disease. J Clin Neurosci 2017; 18: 939-944.

8. She D, Yang X, Xing Z, et al. Differentiating Hemangioblastomas from Brain Metastases Using Diffusion-Weighted Imaging and Dynamic Susceptibility Contrast-Enhanced Perfusion-Weighted MR Imaging. AJNR Am J Neuroradiol 2016; 37: 1844-1850.

9. Essig M, Shiroishi MS, Nguyen TB, et al. Perfusion MRI: The five most frequently asked technical questions. Am J Roentgenol 2013; 200: 24-34.

10. Zimny A, Sasiadek M. Contribution of perfusion-weighted magnetic resonance imaging in the differentiation of meningiomas and other extra-axial tumors: case reports and literature review. J Neurooncol 2011; 103: 777-783.
11. Kang Y, Choi SH, Kim YJ, et al. Gliomas: Histogram analysis of apparent diffusion coefficient maps with standard- or high-b-value diffusion-weighted MR imaging - correlation with tumor grade. Radiology 2011; 261: 882-890.

12. Murakami R, Hirai T, Kitajima M, et al. Magnetic resonance imaging of pilocytic astrocytomas: Usefulness of the minimum Apparent Diffusion Coefficient (ADC) value for differentiation from highgrade gliomas. Acta Radiol 2008; 49: 462-467.

13. Bing F, Kremer S, Lamalle L, et al. Intérêt de l'imagerie de perfusion dans l'étude des astrocytomes pilocytiques et des hémangioblastomes: étude préliminaire. J Neuroradiol 2009; 36: 82-87.

14. She DJ, Xing Z, Zeng Z, et al. Differentiation of hemangioblastomas from pilocytic astrocytomas using 3-T magnetic resonance perfusion-weighted imaging and MR spectroscopy. Neuroradiology 2015; 57: 275-281.

15. Mangla R, Kolar B, Zhu T, et al. Percentage signal recovery derived from MR dynamic susceptibility contrast imaging is useful to differentiate common enhancing malignant lesions of the brain. Am J Neuroradiol 2011; 32: 1004-1010.

16. Quadery F, Okamoto K. Diffusion-weighted MRI of haemangioblastomas and other cerebellar tumours. Neuroradiology 2003; 45: 212-219.

17. de Fatima Vasco Aragao M, Law M, Batista de Almeida D, et al. Comparison of perfusion, diffusion, and $\mathrm{mr}$ spectroscopy between low-grade enhancing pilocytic astrocytomas and high-grade astrocytomas. AJNR Am J Neuroradiol 2014; 35: 1495-1502.

18. Schneider JF, Confort-Gouny S, Viola A, et al. Multiparametric differentiation of posterior fossa tumors in children using diffusion-weighted imaging and short echo-time 1H-MR spectroscopy. J Magn Reson Imaging 2007; 26: 1390-1398. 